\title{
Extragalactic science with ALMA
}

\author{
George J. Bendo ${ }^{1}$ and the UK ALMA Regional Centre Node \\ ${ }^{1}$ UK ALMA Regional Centre Node, Jodrell Bank Centre for Astrophysics \\ School of Physics and Astronomy, University of Manchester \\ Oxford Road, Manchester M13 9PL, United Kingdom \\ email: george.bendo@manchester.ac.uk
}

\begin{abstract}
The Atacama Large Millimeter/submillimeter Array (ALMA) is a telescope comprising 66 antennas that is located in the Atacama Desert in Chile, one of the driest locations on Earth. When the telescope is fully operational, it will perform observations over ten receiver bands at wavelengths from 9.5-0.32 mm (31-950 GHz) with unprecedented sensitivities to continuum emission from cold $(<20 \mathrm{~K})$ dust, Bremsstrahlung, and synchrotron emission as well as submillimetre and millimetre molecular lines. With baselines out to $16 \mathrm{~km}$ and dynamic reconfiguration, ALMA will achieve spatial resolutions ranging from $3^{\prime \prime}$ to $0.010^{\prime \prime}$, allowing for detailed imaging of continuum or molecular line emission from 0.1-1 kpc scale gas and dust discs in highredshift sources or 10-100 pc scale molecular clouds and substructures within nearby galaxies. Science observations started on 30 September 2011 with 16 antennas and four receiver bands on baselines up to $400 \mathrm{~m}$. The telescope's capabilities will steadily improve until full operations begin in 2013 .
\end{abstract}

Keywords. telescopes, submillimeter, instrumentation: interferometers

\section{Introduction}

Science observations by the Atacama Large Millimetre/submillimeter Array (ALMA) started on 30 September 2011. At the time of this writing, ALMA still has limited science capabilities. The telescope has only 16 antennas operational and bands 3,6,7, and 9 available (Mathys 2011). Nonetheless, ALMA is still able to produce both spectral line and continuum maps with sensitivities that are either comparable to or better than other millimetre and submillimetre telescopes currently in operation. When fully operational, ALMA will be the most sensitive millimetre and submillimetre telescope for both continuum and spectral line observations. Specifications of the bands available for use on ALMA are given in Table 1.

The first call for proposals was for Cycle 0, which runs from 30 September 2011 to 30 June 2012. A total of 919 proposals were submitted, with 112 projects being listed as the highest priority observations. The earliest extragalactic targets will include observations of nearby active galactic nuclei including Centaurus A, NGC 1068, and NGC 1097; nearby starburst galaxies such as M83 and NGC 253; infrared luminous galaxies such as Arp 220, NGC 1614, and NGC 6240; gravitational lenses; and high-redshift submillimetre galaxies. In nearby galaxies, the observations will be used to map dust and gas on 10-100 pc scales so as to examine the dynamics and excitation of gas within the centres of the galaxies. For high redshift sources, the observations will be used to identify the optical counterparts of sources and to resolve submillimetre and millimetre gas and dust emission on kpc scales. A complete list of accepted observing proposals is given at http://www.eso.org/public/archives/releases/sciencepapers/eso1137/eso1137.pdf . 
Table 1. ALMA band specifications

\begin{tabular}{|c|c|c|c|c|c|c|}
\hline Band & $\mid \begin{array}{c}\text { Frequency } \\
(\text { GHz })\end{array}$ & $\begin{array}{c}\text { Wavelength } \\
(\mu \mathbf{m})\end{array}$ & $\mid \underset{\left({ }^{\prime \prime}\right)}{\text { Primary Beam }}$ & \begin{tabular}{|} 
Largest Angular \\
Scales (")
\end{tabular} & $\begin{array}{l}\text { Angular } \\
\text { Compact }\end{array}$ & $\begin{array}{l}\text { Resolution }\left({ }^{\prime \prime}\right) \\
\mid \quad \text { Extended }\end{array}$ \\
\hline 1 & $31.3-45$ & $6.7-9.5$ & $145-135$ & 93 & $13-9$ & $0.14-0.1$ \\
\hline 2 & 67-90 & $3.3-4.5$ & $91-68$ & 53 & $6-4.5$ & $0.07-0.05$ \\
\hline $3^{2}$ & $84-116$ & $2.6-3.6$ & $72-52$ & 37 & $4.9-3.6$ & $0.05-0.038$ \\
\hline 4 & $125-163$ & $1.8-2.4$ & $49-37$ & 32 & $3.3-2.5$ & $0.035-0.027$ \\
\hline 5 & $163-211$ & $1.4-1.8$ & $37-29$ & 23 & & \\
\hline $6^{2}$ & $211-275$ & $1.1-1.4$ & $29-22$ & 18 & $2.0-1.5$ & $0.021-0.016$ \\
\hline $7^{2}$ & $275-373$ & $0.80-1.1$ & $22-16$ & 12 & $1.5-1.1$ & $0.016-0.012$ \\
\hline 8 & $385-500$ & $0.60-0.78$ & $16-12$ & 9 & $1.07-0.82$ & $0.011-0.009$ \\
\hline $9^{2}$ & $602-720$ & $0.42-0.50$ & $10-8.5$ & 6 & $0.68-0.57$ & $0.007-0.006$ \\
\hline 10 & $787-950$ & $0.32-0.38$ & $7.7-6.4$ & 3 & $0.52-0.43$ & $0.006-0.005$ \\
\hline
\end{tabular}

Notes:

1 These data are for when ALMA is fully operational. The data are from Observing with ALMA: A Primer for Early Science (Schieven 2010).

${ }_{2}$ These are bands available in Cycle 0 onwards. Other bands will be developed and made available later.

\section{ALMA support}

ALMA is a partnership between North America, Europe, and East Asia in cooperation with the Republic of Chile. Each region has its own ALMA Regional Centre (ARC), with telescope activity coordinated by the Joint ALMA Observatory (http://www.almaobservatory.org/). Additional details on the organisational structure are given by the ALMA Cycle 0 Proposer's Guide (Mathys 2011) and Observing with ALMA: A Primer for Early Science (Schieven 2010). The ARCs provide a wide range of support services for users. First of all, the ARCs provide user support with proposal preparation and submission, including tutorials on using the ALMA Observing Tool, which is used to prepare and submit proposals. For proposals that are accepted for observation, the ARCs will create and evaluate the scheduling blocks, which are used as instructions on performing the observations. Following the completion of observations, the ARCs will provide assistance with data reduction. The ARCs not only provide periodic workshops on using CASA (the data reduction software for ALMA) but also provide one-to-one user support for data processing. The ARCs also work on general software development and engage in public outreach work. The three ARCs are:

- The European Southern Observatory (http://almascience.eso.org/) for Europe. (The European ARC also has nodes in the Czech Republic, France, Germany, Italy, the Netherlands, Sweden, and the United Kingdom that provide local support for ALMA users.)

- The National Radio Astronomy Observatory (https://science.nrao.edu/facilities/ alma) for North America.

- The National Astronomical Observatory of Japan (http://almascience.nao.ac.jp) for East Asia.

\section{References}

Mathys, G. 2011, ALMA Cycle 0 Proposer's Guide (Garching: ESO), Version 1.1

Schieven, G., 2011, Observing with ALMA: A Primer for Early Science, (Victoria: NRC Herzberg Institute of Astrophysics), ALMA Doc. 0.1, ver. 2.2 